\section{FEBRUARY 2006}

National Forest Strategy Coalition

Annual General Meeting

21 February, Victoria.

www.nfsc.forest.ca

Global Challenges: Towards Strategic Solutions - Association of British Columbia Forest Professionals Annual General Meeting. 22-24 February, Victoria. Contact: Alan Fry abcfp58@shaw.ca. www.abcfp.ca/practice_development/continuing_education/forestry_ conference-2006

The Art of Professional Practice 20-24 February, Mattawa, Ontario. Contact: John Pineau, Forest Research Partnership john@canadianecology.ca

\section{MARCH 2006}

Forest Leadership Conference: The Sustainability Challenge 01-02 March, Toronto. www.forestleadership.com

International Precision Forestry Symposium 05-10 March, Stellenbosch, South Africa.

Contact: mgor@sun.ac.za

1st Fire Behaviour and Fuels Conference: Fire Management How to Measure Success 27-30 March, Portland, Oregon. www.iawfonline.org/fuels/overview. shtml

\section{APRIL 2006}

$13^{\text {th }}$ Conference of the conference series: Your Forest - Your Choice 19-20 April, Huntsville, Ontario. www.westwindforest.ca or contact Maxine Davidson at maxinedavidson@westwindforest.ca
College of Alberta Professional

Forest Technologists.

20-21 April, Edmonton.

Contact: Wayne Williams at

registrar@forestechs.ab.ca

Brazil Forestry Study Tour

30 April-07 May

Curitiba, Brazil.

www.worldforestinvestment.com

\section{MAY 2006}

Climate Change Technology

Conference - Engineering Challenges and Solutions in the 21st Century

04-12 May, Ottawa.

Contact: John Grefford at

Grefford@ieee.org

GPS-Radiotelemetry workshop

10-11 May

Selkirk College, Castelgar, B.C.

Contact: Dr. Robert D'Eon at

rdeon@interchange.ubc.ca

\section{JUNE 2006}

$12^{\text {th }}$ International Symposium on Society \& Resource Management Social Sciences in Resource Management: Global Challenges - Local

Responses.

03-08 June, Vancouver.

www.issrm2006.rem.sfu.ca

College of Alberta Professional Foresters Annual General Meeting 22 June, Edmonton.

Contact: Doug Krystofiak at (780) 432-1177

\section{JUly 2006}

$30^{\text {th }}$ Meeting of the Canadian Tree Improvement Association: Canada's Forests - Enhancing Productivity, Protection and Conservation. 24-29 July, Charlottetown, P.E.I. Contact: kathy.tosh@gnb.ca
SEPTEMBER 2006

$10^{\text {th }}$ National Forest Congress: Sustainable Forest Management in the Boreal - A Global Challenge. 24-27 September, Gatineau-Ottawa. 1-866-441-4006 www.nfc-cfn.ca

\section{OCTOBER 2006}

The Lake Abitibi Model Forest and the Canadian Institute of Forestry are proud to host:

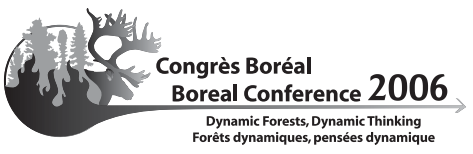

The Boreal Conference 2006 October 1-4, 2006 with optional field trips Oct. 5 \& 6

Cochrane / Moosonee /

Moose Factory, Ontario. www.borealconference2006.ca

Sustainable Forest Management with Fast Growing Plantations.

10-13 October,

Charleston, North Carolina. www.iufro.org/science/divisions/ division-4

Seventh Canadian Urban Forestry Conference - The Tree at the Centre of Urban Development 11-13 October, Quebec City. Contact: Michael Rosen at mrosen@treecanada.ca

\section{MARCH 2007}

2nd Fire Behaviour and Fuels Conference - Fire Behaviour Fundamentals and Applications 26-30 March, San Diego. www.iawfonline.org 